\title{
RESULTADOS PRELIMINARES SOBRE EL COMPORTAMIENTO HIDROLOGICO Y LAS TASAS DE EROSION EN PEQUEÑAS CUENCAS EXPERIMENTALES DE LA CALMA (MONTSENY, BARCELONA)
}

\author{
Cristina Belillas* \\ Ferrán Rodà*
}

\begin{abstract}
RESUMEN
Se comentan las pautas de respuesta hidrológica en una cuenca de landas de 6,7 ha. a partir de los hidrogramas de tres precipitaciones individuales. Cuando los suelos de la cuenca están secos, el avenamiento producido es despreciable. En dos ocasiones en que la cuenca estaba húmeda, el avenamiento atribuible a la crecida representó entre el $45 \%$ y el $85 \%$ de la precipitación total. Estos resultados ponen de manifiesto la importancia de las condiciones hidrológicas antecedentes.

Durante 2,5 años, esta cuenca produjo $21 \mathrm{~kg} . \mathrm{ha}^{-1}$ año-1 de sedimentos de arrastre de fondo. Una cuenca quemada de menor pendiente generó $2 \mathrm{~kg}$. $h a^{-1}$ año ${ }^{-1}$ de arrastre de fondo durante 1 año después del incendio. El fuego no produjo pues erosión significativa mediante arrastre de fondo, pero aún está por evaluar la importancia de otras posibles formas de erosión. La inspección visual de la cuenca quemada permite concluir que debieran extremarse las precauciones al quemar landas en laderas de pendiente superior a $\operatorname{los} 10-15^{\prime \prime}$.
\end{abstract}

\section{SUMMARY}

The hydrological response of a 6.7 ha., heathland-covered watershed in NE Spain during three storm events is discussed. When the soils were dry only negligible runoff was produced. In two ocasions when the catchment 


\section{CRISTINA BELILLAS, FERRAN RODA}

was wet, storm runoff amounted to between $45 \%$ and $85 \%$ of total rainfall. This underlines the role of antecedent soil water condition in determining watershed response.

Bedload transport delivered $21 \mathrm{~kg} . \mathrm{ha}^{-1}$ year-1 of sediment in this watershed. A nearby, burned watershed with gentler relief produced $2 \mathrm{~kg}$. ha-l year-l of bedload transport during the first year after the fire. Burning of heathlands on moderate slopes does not produce significant erosion through bedload transport, but other erosion pathways have not been yet assessed. Upon inspection of the burned watershed, it is strongly recommended that heathlands on slopes steeper than $10-15^{\circ}$ be only burned with special precautions to reduce erosión.

El trabajo que estamos desarrollando en La Calma forma parte del proyecto que han descrito sucintamente Avila \& Rodà (1985). En el sector culminal del macizo de La Calma hemos instrumentado una pequeña cuenca experimental (TM5) sobre substrato impermeable con el fin de conocer los balances anuales de entrada y salida de agua y nutrientes a nivel de cuenca. Uno de los principales objetivos del programa es precisamente comparar el comportamiento hidrológico y biogeoquímico de esta cuenca, situada en una cabecera de relieve moderado y cubierta por landas pastoreadas, con el de la cuenca experimental de encinar montano (TM9, Avila \& Rodà 1985) que se encuentra en una vertiente lateral muy abrupta del mismo macizo, sobre la misma litología, y que está cubierta por un bosque denso no pastoreado. Ambas cuencas disponen, entre otras instalaciones, de una estación de aforo con limnígrafo y pluviógrafo.

Por otra parte, los pastores incendian recurrentemente las landas de La Calma para favorecer el pasto. Hemos creído interesante intentar evaluar las pérdidas de nutrientes y la magnitud de la erosión química y física que esta práctica de manejo pueda ocasionar. A tal efecto iniciamos en octubre 1982 el muestreo del agua de avenamiento de una nueva cuenca (SN), que fue quemada por los pastores en marzo 1983. En julio 1983 instalamos en esta cuenca un vertedero en $\mathrm{V}$ para facilitar los aforos pero, a diferencia de TM5, SN no dispone de limnígrafo.

En este artículo presentamos resultados preliminares sobre: 1) las pautas de respuesta hidrológica de TM5; y 2) las magnitudes del arrastre de fondo en SN después del incendio y en TM5.

\section{Areas de estudio}

TM5 es una cuenca intermitente de 6,7 ha., orientada al NW, a una altitud entre 1.240 y 1.337 m. s.n.m. La pendiente media entre los extremos de la cuenca es de $12^{\circ}$. Las vertientes laterales tienen pendientes entre 14 y $23^{\circ}$. 


\section{EROSION EN CUENCAS EXPERIMENTALES}

La roca madre es una filita cambro-ordovícica y el suelo es un ránker poco profundo. Estimamos que las medias anuales de precipitación y temperatura deben estar alrededor de los $1.000 \mathrm{~mm}$. y los $8^{\circ} \mathrm{C}$, respectivamente. Los dos tercios superiores de la cuenca están cubiertos por una landa alta de la retama Cytisus scoparius, instalada sobre cultivos abandonados hacia 1963 y que no ha sido quemada. El tercio inferior lleva una landa rasa de Calluna vulgaris que no ha sufrido incendios desde antes de 1967. El fondo de valle, relativamente ancho, está ocupado por una franja de prados dominados por el helecho Pteridium aquilinum.

SN es una cuenca intermitente de 20,6 ha. cercana a TM5 pero orientada al SSE, a una altitud entre 1.180 y $1.278 \mathrm{~m}$. La pendiente media entre los extremos de la cuenca es de $7^{\circ}$, bastante menor que en TM5. Además, las vertientes de la cabecera y el tramo central del cauce tienen en SN pendientes muy moderadas $\left(3-8^{\circ}\right)$. En un pequeño sector cerca de la salida de la cuenca las vertientes laterales son muy abruptas $\left(30-38^{\circ}\right)$. La roca madre está formada por filitas y esquistos. Antes del incendio de 1983, una landa de Calluna cubría la mayor parte de SN. El fuego afectó prácticamente a toda la cuenca.

Ambas cuencas están sometidas a pastoreo extensivo de ovejas y vacas.

\section{Métodos}

El caudal de TM5 se obtiene de modo continuo a partir del limnígrafo y de la calibración empírica del vertedero de cresta fina en $V$ de $60^{\circ}$ realizada mediante aforos volumétricos. Un pluviógrafo situado junto a la estación de aforo y conectado al registrador del limnígrafo permite saber de forma sincrónica la intensidad de las precipitaciones.

En TM5 y SN, el material del arrastre de fondo (bedload transport) se recoge periódicamente en el estanque de los respectivos vertederos, y se seca en la estufa a $105^{\circ} \mathrm{C}$ para averiguar el peso seco. Cuando la cantidad de material es excesiva, se secan varias submuestras y se resta su contenido en agua del peso fresco total recogido.

\section{Resultados y discusión}

\section{Respuesta hidrológica de TM5}

En este apartado examinaremos tres hidrogramas obtenidos en TM5 que corresponden a tres precipitaciones individuales. Las características de estas precipitaciones y de sus hidrogramas pueden verse en la Tabla 1 y en las Figuras 1 a 3. 


\section{CRISTINA BELILLAS, FERRAN RODA}

La lluvia del 29 octubre 1983 se produjo en un momento en que los suelos de la cuenca estaban muy secos. El arroyo estaba totalmente seco al empezar la lluvia. La precipitación fue de $41,0 \mathrm{~mm}$., que cayeron de forma continuada y poco intensa (Tabla 1). El nivel del agua en el estanque del vertedero, que inicialmente estaba por debajo del vértice de la $\mathrm{V}$, comenzó a subir cuando habían llovido $11,3 \mathrm{~mm}$., pero no alcanzó el vértice de la $\mathrm{V}$ (y el limnígrafo no empezó a registrar caudales medibles) hasta $8 \mathrm{~h}$. $10^{\prime}$ y 22,8 mm. después de comenzada la lluvia (Fig. 1). El caudal máximo fue de sólo $0,04 \mathrm{~L} \mathrm{~s}^{-1}$. Este caudal se mantuvo durante $3 \mathrm{~h}, 30^{\prime}$, por lo cual el hidrograma presenta forma de meseta (Fig. 1). La recesión fue muy breve. A pesar de que la precipitación fue relativamente abundante, sólo el $0,03 \%$ de la lluvia se transformó en avenamiento.

La lluvia del 1 abril 1984 tuvo lugar cuando la cuenca estaba húmeda o muy húmeda tras la fusión de la nieve caída durante el mes de marzo (148 $\mathrm{mm}$. entre el 28 enero y el 31 marzo). El día 25 de marzo quedaba ya poca nieve en TM5, y el arroyo llevaba un caudal alto $\left(10,3 \mathrm{~L} \mathrm{~s}^{-1}\right)$. El día 1 de abril llovieron $42,8 \mathrm{~mm}$. La intensidad media fue baja y similar a la del 29 octubre, aunque la intensidad horaria máxima fue algo mayor (Tabla 1). El caudal al inicio de la precipitación era de $1,1 \mathrm{~L} \mathrm{~s}^{-1}$, y no comenzó a aumentar hasta que habían llovido $30,3 \mathrm{~mm}$. El caudal máximo fue de $4,9 \mathrm{~L} \mathrm{~s}^{-1}$. El hidrograma presenta nuevamente forma de meseta, pues el caudal máximo se mantuvo por lo menos durante 51 h. 20' (Fig. 2). Una avería del limnígrafo interrumpió el registro durante 62 horas (Fig. 2). La recesión fue larga y gradual. Para calcular el avenamiento total se ha utilizado una reconstrucción hipotética del segmento del hidrograma en que no hubo registro. Las distintas hipótesis plausibles sobre la variación del caudal durante este lapso tienen sólo un efecto moderado sobre la integral del hidrograma (Fig. 2). Con esta reconstrucción, el avenamiento durante el período abarcado por la Figura 2 representó el $72 \%$ de la precipitación total. No sabemos cuál hubiera sido el avenamiento que se hubiera registrado de no haber ocurrido la precipitación del 1 de abril, pues aún no conocemos suficientemente las pautas de recesión de TM5 bajo distintas condiciones hidrológicas. No obstante, bajo la hipótesis maximal de que el caudal inicial se hubiese mantenido constante durante 8 días, el avenamiento atribuible a la crecida habría representado el $45 \%$ de la precipitación. Dicho avenamiento debe pues situarse entre el $45 \%$ y el $72 \%$ de la precipitación total.

A diferencia de los dos casos anteriores, nuestros datos sobre la crecida del 18 mayo 1984 no corresponden a una precipitación individual íntegra. El período que aquí analizaremos comienza a las $13 \mathrm{~h} .17^{\prime}$ de ese día, pero inmediatamente antes habían caído $56,0 \mathrm{~mm}$. durante los cuales el limnígrafo no funcionó. A partir de las $13 \mathrm{~h}$. $17^{\prime}$ 'se recogieron otros $46,8 \mathrm{~mm}$., que cayeron a una intensidad media moderada, aunque la intensidad horaria máxima fue relativamente alta (Tabla 1). El caudal a las 13 h. 17' era muy alto $\left(26,5 \mathrm{~L} \mathrm{~s}^{-1}\right.$; compárese con los caudales máximos de las crecidas anterio- 


\section{EROSION EN CUENCAS EXPERIMENTALES}

res, Tabla 1) debido a la lluvia recién caída. A partir de las $13 \mathrm{~h} .17^{\prime}$, con la nueva lluvia, el caudal aumentó bruscamente hasta un máximo de $90,5 \mathrm{~L}$ $\mathrm{s}^{-1}$. En esta ocasión el hidrograma presenta un pico definido (Fig. 3). La recesión fue larga. El avenamiento total durante el período abarcado por la Figura 3 fue de $91,3 \mathrm{~mm}$. El avenamiento que se hubiera producido de haber cesado la lluvia a las $13 \mathrm{~h}$. 17' puede calcularse aplicando a este instante la recesión obtenida en esta crecida a partir del momento en que el caudal desciende por debajo de $26,5 \mathrm{~L} \mathrm{~s}^{-1}$. Este procedimiento puede justificarse porque, al contrario que el 1 de abril, las condiciones hidrológicas eran similares (muy húmedas o saturadas) en ambos momentos. Como resultado, $51,3 \mathrm{~mm}$. hubieran salido de la cuenca de haber cesado la lluvia y, por lo tanto, 40,0 $\mathrm{mm}$. sería el avenamiento atribuible a la precipitación habida tras las $13 \mathrm{~h}$. 17 ', lo cual representa un coeficiente de escorrentía del $85 \%$.

Como acabamos de ver, la respuesta hidrológica de TM5 fue muy distinta en las tres ocasiones, a pesar de que la cantidad de precipitación resultó muy similar en los tres casos y de que las intensidades fueron del mismo orden de magnitud (Tabla 1). Se pone así de manifiesto la importancia clave de las condiciones hidrológicas antecedentes. En las tres crecidas analizadas tanto el caudal máximo como el porcentaje de avenamiento aumentan de modo notable al aumentar el grado de humedad de la cuenca, hecho que debe interpretarse a la luz del concepto del "área generadora variable" (variable source area, Dunne 1978). Esta concepción considera que, en zonas donde la escorrentía rápida (quickflow) no está dominada por la escorrentía superficial de Horton (agua precipitada a intensidad mayor que la capacidad de infiltración y que llega al cauce escurriendo por la vertiente sin haberse infiltrado), los principales mecanismos que generan escorrentía rápida son el flujo de retorno (agua infiltrada que vuelve a salir a la superficie procedente de un suelo saturado) y la precipitación que cae sobre las áreas saturadas. Sólo las zonas de suelo saturado generarán escorrentía rápida por estos dos mecanismos. El área generadora varía estacionalmente y durante el curso de una precipitación individual porque el área saturada se contrae hacia el tramo inferior del cauce principal a medida que la cuenca se seca, y se expande remontando la red de drenaje y la base de las laderas a medida que la cuenca se humedece. La magnitud y la rapidez de la respuesta hidrológica de una cuenca en un momento dado vendrán determinadas en gran parte por la proporción de la cuenca que ocupe el área generadora.

Un aspecto sorprendente de nuestros datos es la forma de meseta que presenta dos de los tres hidrogramas de TM5 aquí comentados. En particular, no nos atrevemos a descartar la posibilidad de que la dilatada meseta del 1 abril 1984 (Fig. 2) no fuera debida a un mal funcionamiento del limnígrafo. Sin embargo, en una pequeña cuenca de Eucalyptus en Australia, Topalidis \& Curtis (1982) también obtienen frecuentemente hidrogramas en meseta, aunque en los ejemplos que presentan estos autores la duración de la meseta es de 1 a 6 horas. Será necesario disponer de un mayor número de crecidas para caracterizar el comportamiento hidrológico de TM5. 


\section{Arrastre de fondo}

En TM5, los sedimentados depositados por arrastre de fondo durante 2,5 años (6 enero 1982 a 12 julio 1984) representaron $21,1 \mathrm{~kg}$. ha ${ }^{-1}$ año $^{-1}$ (peso seco). En pequeñas cuencas de bosque caducifolio en Hubbard Brook (New Hampshire, USA) se producen en promedio $25,7 \mathrm{~kg} . \mathrm{ha}^{-1} \mathrm{anno}^{-1} \mathrm{de}$ arrastre de fondo (Likens et al. 1977). Durante 2 años, el arrastre de fondo en una cuenca de encinar de $55 \mathrm{ha}$. en Prades (Tarragona) fue de 0,65 kg. ha $^{-1}$ año $^{-1}$ (Lledó \& Escarré 1985). La moderada magnitud de todas estas cifras evidencia el control biótico que las cuencas cubiertas de vegetación ejercen sobre la exportación particulada (Bormann et al. 1969), incluso con pendientes relativamente pronunciadas.

En SN, el arrastre de fondo durante 1 año después del incendio fue de $1,9 \mathrm{~kg}$. ha $\mathrm{hano}^{-1} \mathrm{ano}^{-1}$. No cabe una comparación directa con TM5 porque esta última, aunque no haya sido quemada, no constituye un control de $\mathrm{SN}$, tal y como se explica en la introducción y en la descripción de las áreas de estudio. El arrastre de fondo obtenido en SN es menor que el de TM5 porque las pendientes de las laderas y del cauce son mucho menores en la primera cuenca. De hecho, un año después del fuego, durante el cual hubo precipitaciones copiosas en varias ocasiones, sólo hemos observado en $\mathrm{SN}$ indicios graves de erosión en el pequeño sector de pendientes muy abruptas en la parte inferior de la cuenca. Los resultados demuestran que el incendio total de una landa densa sobre pendientes moderadas no produjo en esta cuenca erosión significativa por arrastre de fondo. Están aún por evaluar las contribuciones de las demás vías erosivas (sedimentos en suspensión, materiales arrastrados de las vertientes que quedan retenidos en el cauce, denudación química) así como las pérdidas de nutrientes producidas por el fuego. No obstante, el estado actual de la cuenca quemada nos permite concluir que debieran extremarse las precauciones al quemar landas situadas en laderas con una pendiente mayor de $10-15^{\circ}$ y que, si es imprescindible quemarlas para regenerar el pasto, el fuego se haga de modo que alternen en la misma ladera franjas quemadas y no quemadas para reducir la erosión.

\section{Agradecimientos}

La Caixa d'Estalvis de Barcelona, el Proyecto Cooperativo 0370 concedido por el Comité Conjunto Hispano-Norteamericano para Asuntos de Cooperación, el Servei del Medi Natural de la Generalitat de Catalunya, y el Servei de Parcs Naturals de la Diputación de Barcelona han contribuido a la financiación de este trabajo. Agradecemos la ayuda que en todo momento nos ha prestado el sr. J.A. Montiel. 
Tabla 1. Características de las precipitaciones y los hidrogramas correspondientes a tres crecidas en una pequeña cuenca de landas (TM5, Montseny, Barcelona)

\begin{tabular}{|l|l|c|c|c|}
\cline { 3 - 5 } \multicolumn{2}{c|}{} & $\begin{array}{c}29 \text { octubre } \\
1983\end{array}$ & $\begin{array}{c}1 \text { abril } \\
1984\end{array}$ & $\begin{array}{c}18 \text { mayo } \\
1984\end{array}$ \\
\hline Precipitación total & $\mathrm{mm}$ & 41,0 & 42,8 & 46,8 \\
Precipit. excl. colas* & $\mathrm{mm}$ & 39,0 & 41,8 & 46,5 \\
Duración excl. colas* & horas & 9,2 & 11,0 & 6,2 \\
Intensidad media excl. colas* & $\mathrm{mm} \mathrm{h}^{-1}$ & 4,3 & 3,8 & 7,5 \\
Intensidad horaria máxima & $\mathrm{mm} \mathrm{h}^{-1}$ & 6,8 & 10,5 & 14,0 \\
Caudal anterior & $\mathrm{L} \mathrm{s}^{-1}$ & 0 & 1,1 & 26,5 \\
Caudal máximo & $\mathrm{L} \mathrm{s}^{-1}$ & 0,04 & 4,9 & 90,5 \\
Avenamiento total** & $\mathrm{mm}^{*}$ & 0,01 & 30,7 & 91,3 \\
Avenamiento atribuible a la crecida & $\mathrm{mm}^{*}$ & 0,01 & $>19$ & 40,0 \\
Avenamiento/Precipitación *** & $\%$ & 0,03 & $45-72$ & 85 \\
Tiempo de crecida & horas & 4,5 & 10 & 1,2 \\
Tiempo de retardo & horas & 1,3 & 9 & $<0,5$ \\
Estado antecedente & & seco & húmedo o & muy húmedo \\
& & & muy húmedo & 0 saturado \\
\hline
\end{tabular}

* Se han excluido los períodos iniciales y finales con una intensidad inferior a $1 \mathrm{~mm} . \mathrm{h}^{-1}$.

* Avenamiento registrado durante los períodos abarcados por las Figuras 1 a 3.

*** Cociente entre el avenamiento atribuible a la crecida y la precipitación total. 
CRISTINA BELILLAS, FERRAN RODA

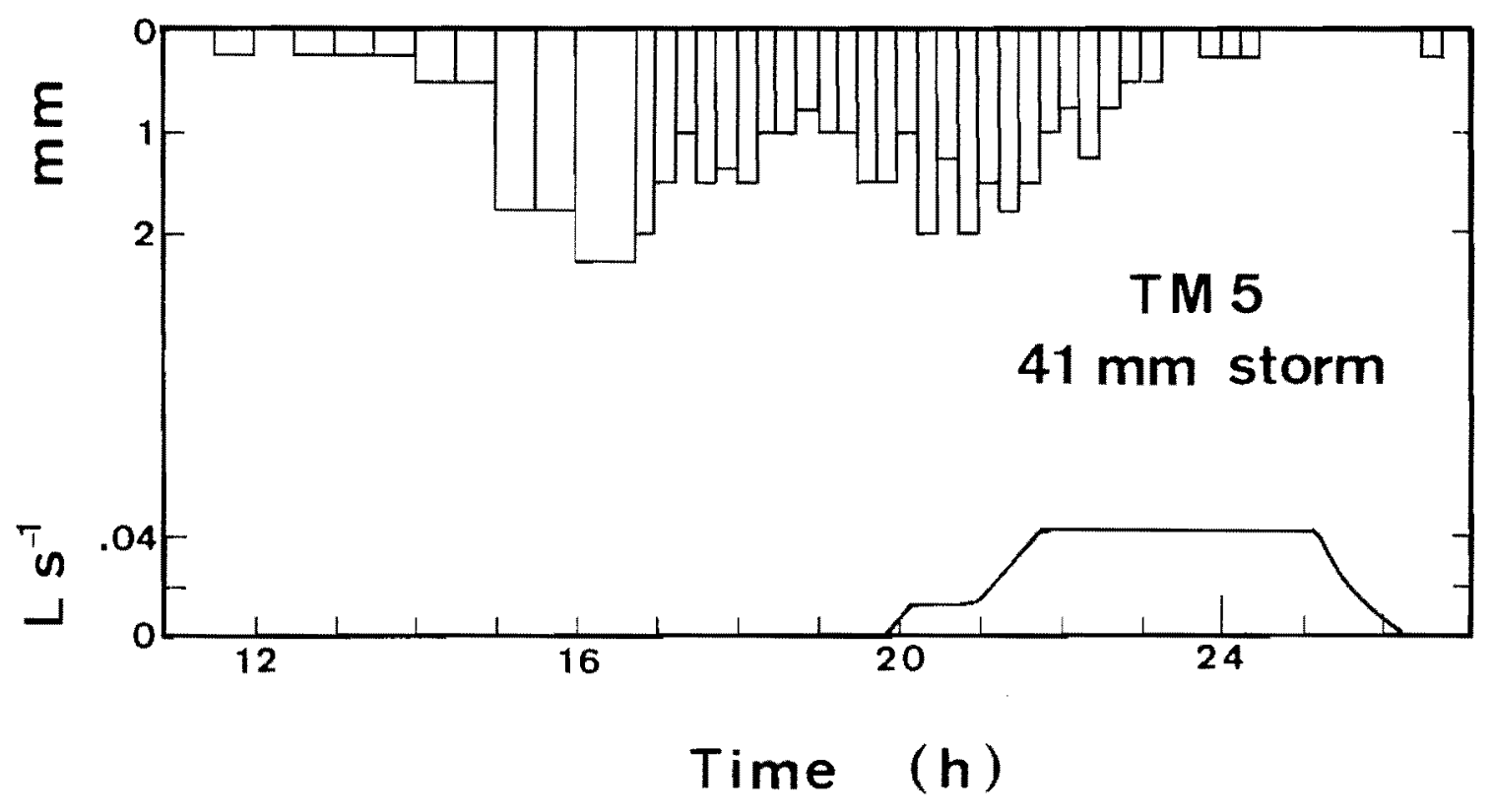

Figura 1.

Crecida del 29 octubre 1983. 


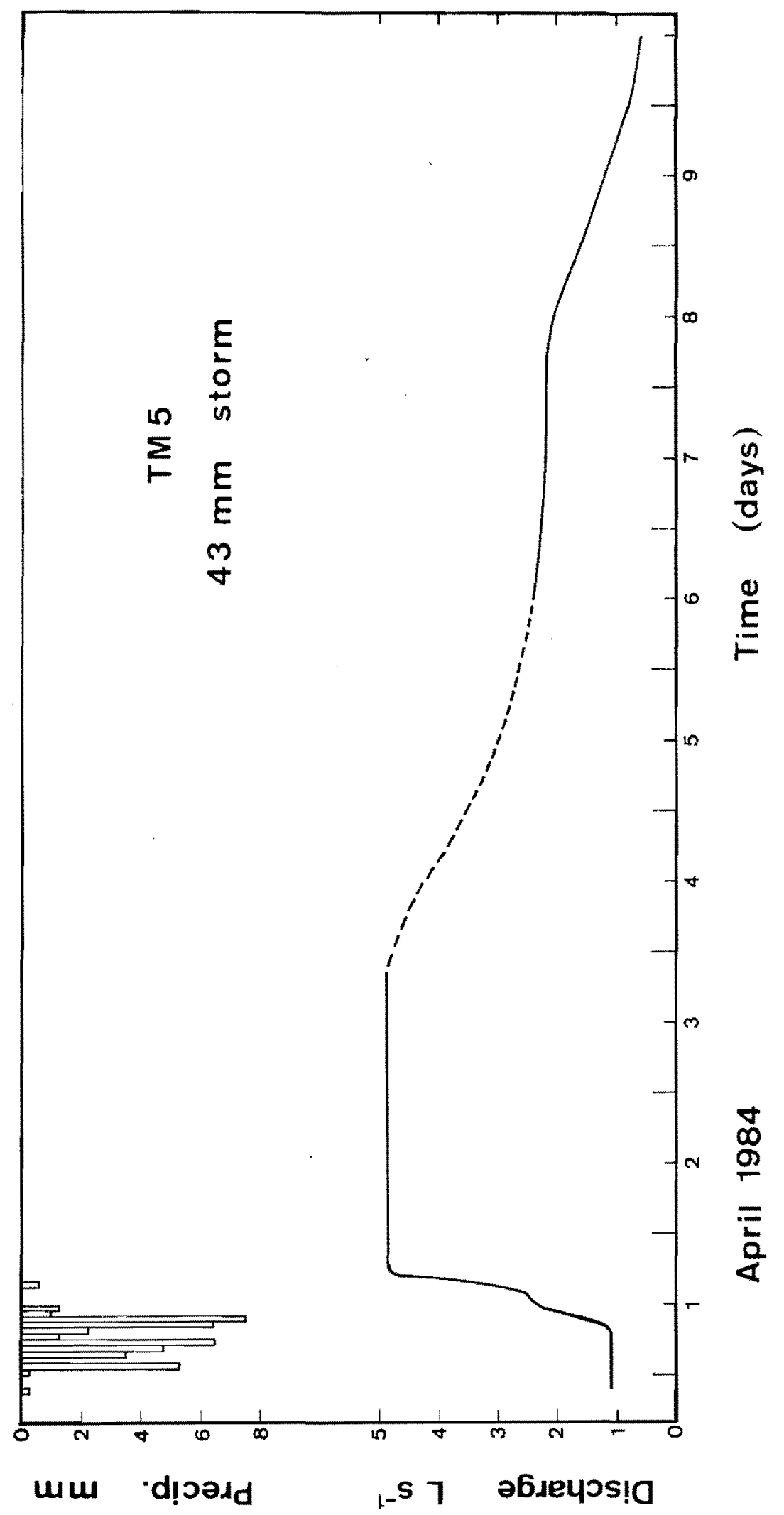

Figura 2.

Crecida del 1 abril 1984. 
CRISTINA BELILLAS, FERRAN RODA

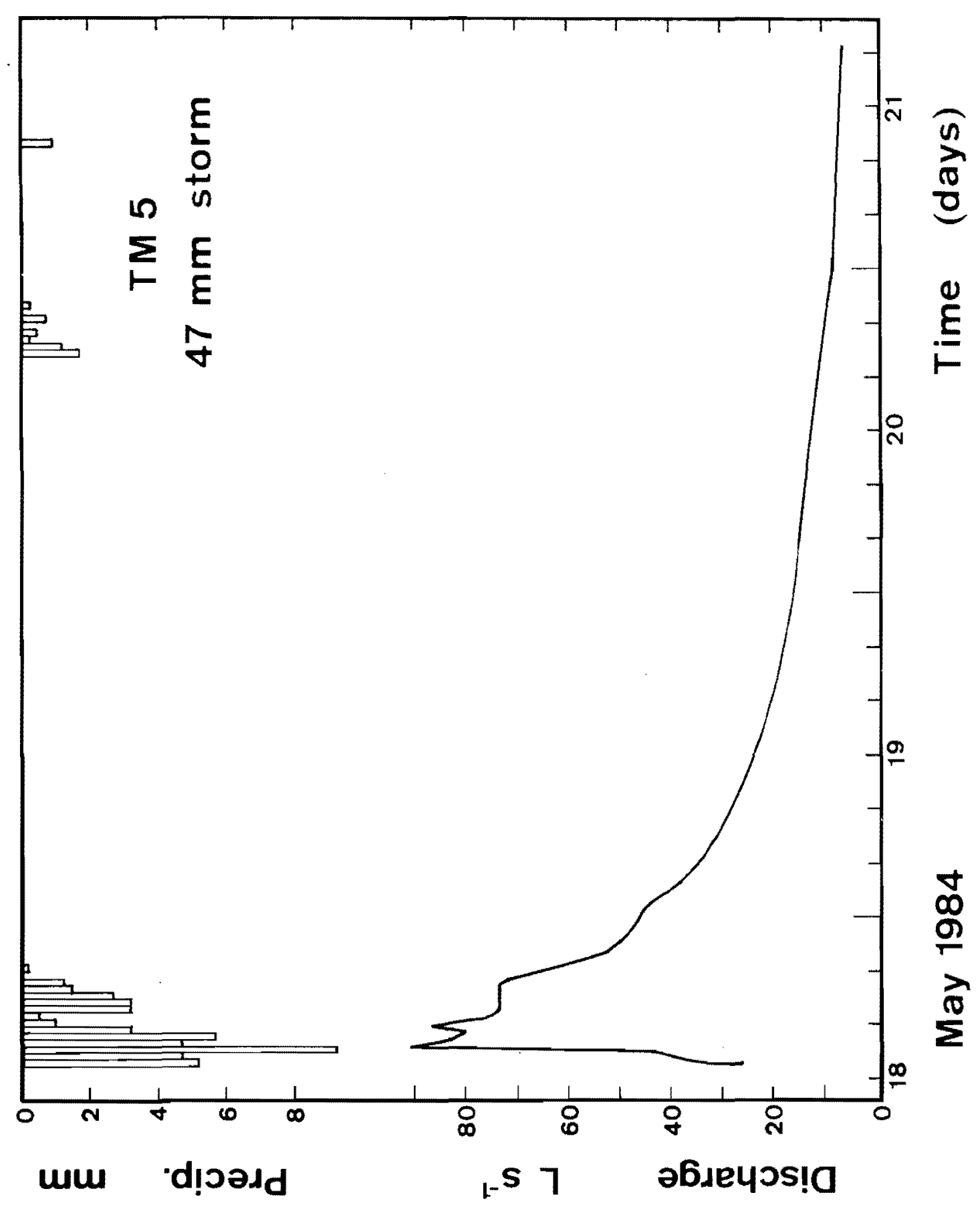

Figura 3.

Crecida del 18 mayo 1984. 


\section{EROSION EN CUENCAS EXPERIMENTALES}

\section{Referencias}

AVILA, A. \& RODA, F., 1985. Variaciones del quimismo del arroyo durante las crecidas en una cuenca de encinar montano. Cuadernos de Investigación Geográfica, 11, Logroño.

BORMANN, F.H., LIKENS, G.E. \& EATON, J.S., 1969. Biotic regulation of particulate and solution losses from a forested ecosystem. BioScience, 19: 600-610.

DUNNE, T., 1978. Field studies of hillslope flow processes. In: M.J. Kirkby (ed.) 'Hillslope Hydrology', pp. 227-293. Wiley. Chichester.

LIKENS, G.E., BORMANN, F.H., PIERCE, R.S., EATON, J.S. \& JOHNSON, N.M., 1977. Biogeochemistry of a Forested Ecosystem. Springer-Verlag. New York.

LLEDO, M.J. \& ESCARRE, A., 1985. Datos preliminares de la exportación de materia disuelta y particulada en una cuenca de encinar. Cuadernos de Investigación Geográfica, 11, Logroño.

TOPALIDIS, S. \& CURTIS, A.A., 1982. The effect of antecedent soil water conditions and rainfall variations on runoff generation in a small eucalypt catchment. In: E.M. O'Loughlin \& L.J. Bren (eds.) 'The First National Symposium on Forest Hydrology', pp. 43-49. The Institution of Engineers. Barton, Australia. 
